\title{
Safer and Healthier Workplaces
}

It was a pleasure to serve as guest editor of this special issue of WORK: A Journal of Prevention, Assessment and Rehabilitation that focuses on "Safer and Healthier Workplaces". This issue is dedicated to workers all over the world who have suffered an accident at work or have acquired an occupational or work-related disease.

Workers should be protected from occupational risks they could be exposed to. This could be achieved through a risk management process, which involves risk analysis, risk assessment and risk control practices. In order to carry out an effective risk management process, it is necessary to have a clear understanding of legal frameworks, concepts, risk analysis, assessment and control processes and the role played by all involved in the process. It is also desirable to base risk management on solid and tested methodologies. Risk management should include the identification and evaluation of risks to work accidents as well as risks to occupational or work-related diseases.

This special issue of WORK offers an array of articles that provide an interesting overview across different applications of current research concerning practices and methodologies related with safety and ergonomics presentedby differentexpert authors.

The first research article authored by Lima and Coelho presents a practical approach to risk assessment of work related musculoskeletal disorders of the upper limbs and neck. The article focus on a case study related with the prevention of musculoskeletal disorders in office work. Musculoskeletal disorders covering a broad range of health problems continue to represent one of the biggest occupational problems in companies. Despite the variety of efforts to control them, including engineering design changes, organizational modifications and working methods' training programs, work related musculoskeletal disorders account for a huge amount of human suffering and economic costs to companies and to healthcare systems.
Jacinto and collaborators present an overview of occupational accident's notification systems within the enlarged European Union. They describe a comparative analysis of the official forms used for reporting occupational accidents within the 27 member-states of the European Union. The importance of this study resides in the fact that statistical data on accidents at work is a relevant source of information in research work as well as to design economic and prevention policies.

Arezes and collaborators introduce a paper on manual materials handling. In this article the authors describes the results obtained from a survey carried out among Portuguese Health and Safety practitioners regarding the characteristics of their companies concerning the existence of manual materials handling and their use of and knowledge about risk assessment methods for manual materials handling tasks. Manual materials handlingtasks are one of the most frequent occupational tasks and due to their characteristics they can have great influence on the development of musculoskeletal disorders.

As ageing occurs, the physical and mental capacity of workers tends to deteriorate, which means that the work ability tends to decrease with the ageing process. The assessment of the work ability of ageing workers is fundamental to make workplaces age-friendly and safe. Nunes and collaborators present a case study with IT workers using the Work Ability Index tool designed to perform such assessment. Based on the obtained results ergonomic measures for improving the work ability of workers were identified and implemented.

Nowadays it is recognized that human behavior can have a crucial impact on the occurrence of accidents at work and occupational diseases. The last paper from Kuhland and Dobernowsky tackle this issue. They present the application of the PIMEX method which allows the worker to observe himself and change the work process repeatedly until satisfactory results are achieved. Therefore, employees are motivated to 
change their working conditions and optimize preventive measures.

I hope this special issue will encourage WORK readers to pursue the challenge of transforming workplaces into safe and healthy places to work.

I would like to thank Dr. Karen Jacobs for the invitation to be a guest editor and many thanks to Victoria Hall for organizing the special issue. I would also like to thank the authors and other persons who helped and encouraged me to make this special issue a reality.
Cheers,

Guest Section Editor Isabel L. Nunes, M.S., Ph.D.

Faculdade de Ciencias e Tecnologia/Universidade Nova de Lisboa

Departamento de Eng. Mecanica e Industrial

Campus de Caparica 2829-516 Caparica

Portugal

E-mail: imn@fct.unl.pt 